\title{
OBSERVATION OF DOUBLE J STENT BACTERIAL COLONISATION AND ITS CORRELATION WITH BACTERIURIA FREQUENCY
}

\author{
Anshul Garg1, Kumar Gaurav Mishra², Pawan Kumar Bharti³, Kanika Goel4, Urvashi Mishra ${ }^{5}$ \\ ${ }_{1}^{1}$ M.Ch. Final Year Trainee, Department of Urology, IGIMS, Patna. \\ ${ }_{2}^{2}$.Ch. $2^{\text {nd }}$ Year Trainee, Department of Urology, IGIMS, Patna. \\ ${ }^{3}$ Senior Resident, Department of Urology, IGIMS, Patna. \\ ${ }^{4}$ Consultant Radiologist, Agra. \\ 5Junior Consultant, Mahavira Vatsalya Hospital., Patna.
}

ABSTRACT

\section{BACKGROUND}

Stents and catheters are widely used in Urology. In this study, we identified the incidence of DJ stent bacterial colonisation and its correlation with bacteriuria frequency.

\section{MATERIALS AND METHODS}

Between January 2015 and June 2016, 170 patients (17 to 72 years old) who underwent DJ stenting were enrolled in the study. Surgeries prior to stenting included PCNL, URS, Pyeloplasty, Pyelolithotomy and Ureterolithotomy. Prior to stenting, sterile urine samples were obtained, and urinary cultures were performed upon removal of the DJ stents along with stent culture. Eighty-three stents were inserted into the right ureter and eighty-seven into the left ureter of the patients.

\section{RESULTS}

Cultures showed bacterial colonisation in 27 (16\%) cases. There was no significant association between positive stent culture and patient age, sex or stent laterality. The rate of colonisation was $2.2 \%, 2.9 \%$, and $25 \%$ when indwelling time was less than 4 weeks, 4-6 weeks, and more than 6 weeks, respectively. In the present study, the rate of infection associated with a DJ stent and urinary infection was not very high. However, bacterial colonisation increases significantly with indwelling time of the stent and sterile culture of urine does not rule out colonisation of the stent.

\section{CONCLUSION}

Bacteriological investigation showed very low rates of colonisation within 6 weeks after the insertion of stents, indicating that ureteral stents can be used safely within that time period. Bacterial colonisation is associated with increased chances of bacteriuria frequency.

\section{KEYWORDS}

DJ Stents, Colonisation, Bacteriuria.

HOW TO CITE THIS ARTICLE: Garg A, Mishra KG, Bharti PK, et al. J. Observation of double J stent bacterial colonisation and its correlation with bacteriuria frequency. J. Evolution Med. Dent. Sci. 2017;6(84):5861-5864, DOI: 10.14260/jemds/2017/1273

\section{BACKGROUND}

As the field of Endourology has developed, a great variety of foreign bodies have been designed, and with the increasing number of biomaterial devices used in Urology, biofilm formation and device infection are issues of growing importance.[1] In the present study, the frequency of colonisation on double J (DJ) stents and stent-associated bacteriuria was investigated. We aimed to compare the relationship between the colonisation of the bladder urine and that on the DJ stents that have been inserted for various reasons. We investigated the importance of indwelling time for infection and antimicrobial susceptibility pattern of isolates in order to establish data on the aetiologic agents of Colonised stents and evaluate the significance of urinary cultures for the identification of colonising microorganisms.

\footnotetext{
'Financial or Other Competing Interest': None.

Submission 28-08-2017, Peer Review 07-10-2017,

Acceptance 12-10-2017, Published 19-10-2017.

Corresponding Author:

Dr. Kumar Gaurav Mishra,

Room No. 31,

IGIMS Campus, Rajabazar, Patna.

E-mail: gauravmishra.purch@gmail.com

DOI: $10.14260 /$ jemds $/ 2017 / 1273$

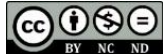

\section{MATERIALS AND METHODS}

This study is a case series of consecutive patients following urologic surgeries. A total of 170 patients from our institution were enrolled in this study between January 2015 and June 2016 after the approval of the institutional review board. All patients received a single dose of prophylactic antibiotics $(1 \mathrm{~g}$ IV cefazolin) prior to the surgery. None of the patients had any of the following three comorbidities: diabetes mellitus, chronic renal disease or immune suppression. Patients with kidney stones (staghorn stones) or presenting with acute pyelonephritis were excluded, because they might have a higher probability of DJ bacterial colonisation and affect the results of the study. The silicone stents were implanted and retained for periods of between 14 days and 72 days, with a mean duration of stent retention of 27 days.

The proportion of stents in the right and left ureters was similar: $82(48 \%)$ and 88 (52\%), respectively. Midstream urine from all patients requiring DJ stent insertion was investigated microbiologically prior to stent insertion and on the day of stent removal. Stents were removed under sterile conditions with the help of a cystoscope and foreign body forceps. Prior to the removal of the stent, bladder urine was collected in a sterile container.

The removed stents were divided into three parts: top, middle, and bottom; 2-3 cm pieces from each part were taken 
for bacteriological investigation. The processed segments of the catheter were placed in sterile test tubes. To wash out the intraluminal part of the catheter and isolate only microorganisms attached to the inner surface of the catheter, $1 \mathrm{~mL}$ sterile Tryptic Soy Broth solution was injected into the inner surface of the catheter segments with a syringe. Then, the liquid culture medium was vortexed for 1 minute, to enable the detection of microorganisms attached to the outer surface of the catheter segment.

Next, $100 \mathrm{~mL}$ of broth was taken separately from tubes containing undiluted and diluted (1/100) samples, and inoculated onto blood agar and eosin methylene blue agar. Plates were incubated for 48 hours at $37^{\circ}$ Celsius. The microorganisms that grew on the agar were evaluated quantitatively (growth of $>1000$ colony-forming units $/ \mathrm{mL}$ was considered significant). Bacteria were identified by the conventional method. All microbial isolates were tested for their susceptibility to a panel of 14 antibiotics.

The Student $t$ and Chi-square tests were used for statistical analysis. The level for statistical significance was based on $p=0.05$, and results were considered statistically significant when $\mathrm{p}<0.05$. All analyses were performed using SPSS version 15 for Windows.

\section{RESULTS}

A total of 170 consecutive patients (92 male and 78 female) were included in the study. Mean patient age was 43.1 years (range 21-73 years). Stone extraction/lithotripsy (n 153), pyeloplasty (n 4), and URSL (n 13) were the indications. The time length of the surgical procedures varied from 11 minutes to 87 minutes, but the correlation between surgery time and colonisation was not investigated. Patients with comorbidities such as diabetes mellitus, chronic renal diseases, or immune suppression were excluded from the study, but hypertension was a comorbidity in 21 study patients. Indications for DJ ureteral stents were oedema and stone fragmentation in 114 patients, stricture in 38 patients and gross hydronephrosis in 18 patients. In our study group, colonisation was detected in 12 female $(7.1 \%)$ and 15 male (8.3\%) patients. The difference was statistically insignificant. All of the colonised stents were from patients who had undergone stone extraction/lithotripsy procedures. No more than one microorganism was detected on any of the stents. There was no relationship between stent colonisation and age or gender $(\mathrm{p}>0.05)$. Colonisation was detected in all parts (top, middle, and bottom) of the outer surface of the DJ stents. The intraluminal parts of the stents were all sterile. Mean indwelling times differed significantly between patients with sterile and non-sterile catheters (Table 1). The rate of colonisation was $2.2 \%, 2.9 \%$, and $25 \%$ when indwelling time was less than 4 weeks, 4- 6 weeks, and more than 6 weeks, respectively (Table 2). Urine culture detected colonisation in only three (11\%) of the 27 colonised patients.

In that patient Pseudomonas was the species cultured from urine as well as from stent. Of the other twenty-four colonised patients, urine samples taken from their bladder were sterile.

\begin{tabular}{|c|c|c|c|c|}
\hline \multirow{2}{*}{ Characteristics } & $\begin{array}{c}\text { Median Age of } \\
\text { the Patient }\end{array}$ & $\begin{array}{c}\text { Indwelling } \\
\text { Time (d) }\end{array}$ & Female & Male \\
\hline Sterile, N (\%) & $41.3(21-69)$ & $22(14-59)$ & 66 & 77 \\
\hline $\begin{array}{c}\text { Nonsterile N } \\
(\%)\end{array}$ & $45.5(28-73)$ & $44(20-72)$ & 12 & 15 \\
\hline Overall & $43.1(21-73)$ & $32(14-72)$ & 78 & 92 \\
\hline P & $>0.05$ & $<0.05$ & & \\
\hline \multicolumn{4}{|c|}{$\begin{array}{c}\text { Table 1. Characteristics of Colonised } \\
\text { and Non-Colonised Stents }\end{array}$} \\
\hline
\end{tabular}

\begin{tabular}{|c|c|c|c|}
\hline $\begin{array}{c}\text { Indwelling } \\
\text { Time (Week) }\end{array}$ & $\mathbf{N}$ & $\begin{array}{c}\text { Colonisation } \\
\text { of Stents (\%) }\end{array}$ & Bacterial Colony \\
\hline$<4$ & 57 & $1(2.2)$ & E. coli (n=1) \\
\hline $4-6$ & 75 & $3(2.9)$ & $\begin{array}{c}\text { E. coli (n=2) } \\
\text { Pseudomonas (n=1) }\end{array}$ \\
\hline$>6$ & 38 & $10(25)$ & $\begin{array}{c}\text { E. coli (n=4) } \\
\text { Pseudomonas (n=3) } \\
\text { Coagulase negative } \\
\text { Staphylococci (n=2) } \\
\text { Acinetobacter (n=1) }\end{array}$ \\
\hline \multicolumn{3}{|c|}{ Table 2. Correlation between Indwelling } \\
Time and Colonisation
\end{tabular}

\section{DISCUSSION}

The DJ ureteral stent has become an integral part of the urological armamentarium. It allows good urinary drainage from the kidney to the bladder, and is generally safe and well tolerated. However, various complications may occur with short- or long-term use of indwelling stents, which vary from minor side effects such as flank and suprapubic pain, haematuria, dysuria, and frequency, to major complications such as vesicoureteric reflux, stent migration, encrustation, stent fracture, and urinary infection. ${ }^{[2]}$ Infections may vary substantially, from a subclinical infection to death due to sepsis. In order to decrease the infection rates of ureteral stents and urinary catheters, different materials have been used such as silicone, polyethylene, polyurethane, biodegradable materials, and drug delivery materials, as well as coatings such as silver, heparin, polytetrafluoroethylene, phosphoryl choline biocides or antibiotics.[3] However, management of the materials associated with biofilm-based infection remains problematic. A biofilm is usually formed by a mixed population of rapidly growing and slowly or nongrowing bacteria. It is an irreversibly encapsulated structured community of microorganisms within a selfdeveloped polymeric matrix that is able to adhere to various biotic and abiotic surfaces. Systemic antibiotic therapy is effective in eliminating circulating bacteria, but it usually fails to protect the surfaces of materials from colonisation, leaving the patient at continued risks of complications or recurrence. [4-7] In several series, it has been shown that an indwelling DJ ureteral stent carries a significant risk of bacteriuria and stent colonisation. The relationship between urine and stent cultures is not clear, although Lojanapiwat[8] published urine culture results indicating colonisation in about two to three of the patients. Klis et al[9] pointed out a great discrepancy between urine and catheter cultures. They concluded that DJ stent retention in the urinary tract is associated with a high risk of bacterial colonisation, whereas the risk of urine infection is much lower. 
In our study, the colonisation rate was $10 \%$, and eight of these colonies (80\%) included Gram-negative bacteria. The high recovery rate of Gram-negative organisms may indicate a preferential adhesion of these bacteria to the biomaterial surface. Of note, when interpreting our study, urine culture is not a particularly sensitive means of detecting stent colonisation; therefore, a negative culture does not rule out a colonised stent. Recently, it was demonstrated that minutes after insertion of a catheter, depositions of host urinary components onto the catheter surface form a conditioning film supporting the bacterial adhesion process actively.[10] Although we did not observe this, several other studies have also demonstrated the ability of uropathogens such as E. coli, Proteus mirabilis, Staphylococcus epidermidis, and Enterococcus faecalis to adhere to, and form biofilms on, ureteral stents within 24 hours. [3,10] Different approaches are being investigated for preventing biofilm formation, and some promising results have been obtained. However, an ideal method has not yet been developed. Future research must aim at identifying effective mechanisms for controlling biofilm formation, such as the application of alternating microcurrent densities on platinum electrodes, use of a selfregenerative surface that removes the conditioning film actively, and development of antimicrobial agents that are effective against bacteria in biofilms.[9] Some urease inhibitors have also been shown to have possible clinical applications in the prevention of catheter encrustation and blockage.[11] In another approach, Elayarajah et al[12] impregnated the stent pieces in an anti-infective solution (a mixture of norfloxacin-metronidazole and a polymer) for uniform surface coating (drug-carrier-coated stents). After coating, an agar diffusion test was performed as a qualitative test of the sensitivity of coated stents against clinical isolates. Quantitative testing revealed that the number of bacteria adhering to the surface of coated stents was reduced significantly.

Kehinde et al[13] showed that the risk of bacteriuria and colonisation of the J stent tip is enhanced significantly by a longer duration of stent retention, female gender, and systemic diseases such as diabetes mellitus, chronic renal failure and diabetic nephropathy and concluded that these categories of patients should undergo shorter stent retention, antimicrobial prophylaxis, and careful follow-up to minimise infectious complications. Coskun et al[14] stressed that early removal of the stent, 2 weeks after renal transplantation, decreased the rate of urinary tract infections. Our study yielded similar results: the longer the duration of stenting, the higher the rate of colonisation $(2.2 \%$ for stents left for $<4$ Weeks vs. $27.9 \%$ for those left for $>4$ weeks). None of our patients had any systemic diseases, except hypertension, precluding the examination of any correlation between such pathologies and colonisation in this study. Our study was potentially limited by its observational design, small sample size, and prophylactic antibiotic treatment of patients, which may have affected bacterial flora. Despite these limitations, our study presents a picture of colonisation of DJ stents under realistic medical conditions, enabling more informed decision making when considering the use of these stents. The findings also indicated that unlike biofilm formation on many other prosthetic implants, colonisation of ureteral stents does not necessarily coincide with the development of symptomatic infection. Recently, Uvin et al[15] investigated the relationship between microbial ureteral stent colonisation and symptoms in children, and showed that nearly half of 199 children had positive stent cultures without any stent related tract symptoms and major side effects. It was also reported by Uvin et al[15] that the rate of urinary tract infection during the first 6 weeks after ureteral reimplantation using indwelling ureteral stents was only $4.6 \%$. Our results were similar, with a colonisation rate of $5.1 \%$ within 6 weeks. We concluded that the clinical significance of bacterial colonisation of an indwelling ureteral stent is low, and also bacteriological investigation showed very low rates in 6 weeks' time; therefore, ureteral stents appear to be safe if used within that time period. Guidelines for the prevention of catheter-associated urinary tract infections were developed over the past decades by clinicians and are still valid. They can now be better understood taking into consideration new techniques such as the utilisation of anti-infective solution based stents, application of alternating microcurrent densities on the instruments, or use of different materials. As overuse of urethral catheters and noncompliance with their recommended use are still apparent, educational and surveillance programs are needed to help maintain good standards of care.[16] Further prospective studies are needed to determine the optimal duration of DJ stent use after different urological approaches. However, we recommend that patients with DJ ureteral stents who can be at risk for bacteraemia be covered by broad-spectrum antibiotics, especially if indwelling time is more than 6 weeks and that the stents should be kept indwelling for the shortest possible time. If a patient with a stent develops a symptomatic infection, antibiotic therapy, if not given earlier, that covers Gram-negative as well as Gram-positive species should be started. The stent should be removed promptly when no longer needed and changed periodically if chronic indwelling is required. Because only a small percentage of urinary cultures identified all colonising microorganisms correctly and as there was a great discrepancy between urine and catheter cultures, removal and bacteriologic evaluation of ureteral stents may be necessary.

\section{CONCLUSION}

Bacteriological investigation showed very low rates of colonisation within 6 weeks after the insertion of stents, indicating that ureteral stents can be used safely within that time period. Bacterial colonisation is associated with increased chances of bacteriuria frequency.

\section{REFERENCES}

[1] Tenke P, Ko"ves B, Nagy K, et al. Update on biofilm infections in the urinary tract. World J Urol 2012;30(1):51-7.

[2] Herrador VJ, Revilla BFJ, Alba AJ, et al. Double J ureteral catheter. Clinical complications. Arch Esp Urol 1998;51(4):361-73.

[3] Stickler DJ. Bacterial biofilms in patients with indwelling urinary catheters. Nat Clin Pract Urol 2008;5(11):598-608.

[4] Reid G, Denstedt JD, Kang YS, et al. Microbial adhesion and biofilm formation on ureteral stents in vitro and in vivo. J Urol 1992;148(5):1592-4. 
[5] Costerton JW, Stewart PS, Greenberg EP. Bacterial biofilms: a common cause of persistent infections. Science 1999;284(5418):1318-22.

[6] Vlastarakos PV, Nikolopoulos TP, Maragoudakis P, et al. Biofilms in ear, nose and throat infections: how important are they? Laryngoscope 2007;117(4):66873.

[7] Donlan RM, Costerton JW. Biofilms: survival mechanisms of clinically relevant microorganisms. Clin Microbiol Rev 2002;15(2):167-93.

[8] Lojanapiwat B. Colonization of internal ureteral stent and bacteriuria. World J Urol 2006;24(6):681-3.

[9] Klis R, Korczak-Kozakiewicz E, Denys A, et al. Relationship between urinary tract infection and selfretaining double-J catheter colonization. J Endourol 2009;23(6):1015-9.

[10] Gabi M, Hefermehl L, Lukic D, et al. Electrical microcurrent to prevent conditioning film and bacterial adhesion to urological stents. Urol Res 2011;39(2):81-8.
[11] Morris NS, Stickler DJ. The effect of urease inhibitors on the encrustation of urethral catheters. Urol Res 1998;26(4):275-9.

[12] Elayarajah, Rajendran $\mathrm{R}$, Venkatrajah, et al. Biodegradable tocopherol acetate as a drug carrier to prevent ureteral stent-associated infection. Pak J Biol Sci 2011;14(5):336-43.

[13] Kehinde EO, Rotimi VO, Al-Awadi KA, et al. Factors predisposing to urinary tract infection after J ureteral stent insertion. J Urol 2002;167(3):1334-7.

[14] Coskun AK, Harlak A, Ozer T, et al. Is removal of the stent at the end of 2 weeks helpful to reduce infectious or urologic complications after renal transplantation? Transplant Proc 2011;43(3):813-5.

[15] Uvin P, Van Baelen A, Verhaegen J, et al. Ureteral stents do not cause bacterial infections in children after ureteral reimplantation. Urology 2011;78(1):154-8.

[16] Liedl B. Catheter-associated urinary tract infections. Curr Opin Urol 2001;11(1):75-9. 